\title{
Periodic solutions for nonlocal $p(t)$-Laplacian systems
}

\section{Shengui Zhang ${ }^{1 *}$ (D)}

\section{"Correspondence:}

zhangshengui315@163.com

${ }^{1}$ College of Mathematics and

Computer Science, Northwest

Minzu University, Lanzhou, People's

Republic of China

\section{Springer}

\begin{abstract}
The purpose of this paper is to investigate the existence of periodic solutions for a class of nonlocal $p(t)$-Laplacian systems. When the nonlinear term is $p^{+}$-superlinear at infinity, some new solvability conditions of nontrivial periodic solutions are obtained by using a version of the local linking theorem. A major point is that we ensure compactness without the well-known Ambrosetti-Rabinowitz type superlinearity condition. In addition, by applying the saddle point theorem, we established the existence of at least one periodic solution for such problems with a $p^{-}$-subquadratic potential.
\end{abstract}

MSC: $34 \mathrm{~J} 37 ; 58 \mathrm{E} 05 ; 70 \mathrm{H} 05$

Keywords: Variational methods; $p(t)$-Laplacian systems; Nonlocal problems; Critical point; Periodic solution

\section{Introduction and main results}

Consider the non-autonomous second order Hamiltonian systems

$$
\left\{\begin{array}{l}
\ddot{u}(t)+\nabla V(t, u(t))=0, \\
u(0)-u(T)=\dot{u}(0)-\dot{u}(T)=0
\end{array} \quad \text { a.e. } t \in[0, T]\right.
$$

where $T>0, u(t) \in \mathbb{R}^{N}, \nabla V(t, u)$ denotes the gradient of $V(t, u)$ in $u$.

In the classical monograph [1], Mawhin and Willem investigated the existence of periodic solutions for problem (1.1) via critical point theory. During the past two decade, inspire by [1] the existence and multiplicity of periodic solutions for systems (1.1) has been studied extensively.

Problems with variable exponent growth conditions arise in the description of the physical phenomena with "pointwise different properties" which first arose from the nonlinear elasticity theory; see [2]. It was also observed that non-homogeneous $p(t)$-Laplacian operators are related to modeling of so-called electrorheological fluids; see [3, 4] for more details of the physical aspects. Another field of application of $p(t)$-Laplacian systems is image processing $[5,6]$. The variable nonlinearity is used to eliminate possible noise and outline the borders of the true image.

(c) The Author(s) 2019. This article is distributed under the terms of the Creative Commons Attribution 4.0 International License (http://creativecommons.org/licenses/by/4.0/), which permits unrestricted use, distribution, and reproduction in any medium, provided you give appropriate credit to the original author(s) and the source, provide a link to the Creative Commons license, and indicate if changes were made. 
In [7-15], the authors studied the existence of periodic solutions, subharmonic solutions and homoclinic orbits for the $p(t)$-Laplacian systems

$$
\left\{\begin{array}{l}
\frac{d}{d t}\left(|\dot{u}(t)|^{p(t)-2} \dot{u}(t)\right)+\nabla V(t, u(t))=0, \quad \text { a.e. } t \in[0, T] \\
u(0)-u(T)=\dot{u}(0)-\dot{u}(T)=0,
\end{array}\right.
$$

where $p(t) \in C\left([0, T], \mathbb{R}^{+}\right)$, the operator $\frac{d}{d t}\left(|\dot{u}(t)|^{p(t)-2} \dot{u}(t)\right)$ is said to be $p(t)$-Laplacian. The $p(t)$-Laplacian operator possesses more complicated nonlinearity than that of the $p$ Laplacian operator, where $p>1$ is a constant. For example, it is inhomogeneous, which provokes some mathematical difficulties. We point out that commonly known techniques for studying constant exponent equations fail in the setting of problems involving variable exponents.

In this paper, we consider the following nonlocal $p(t)$-Laplacian systems:

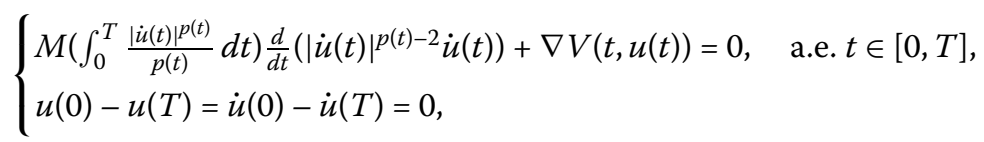

where $T>0, M(s):[0,+\infty) \rightarrow(0,+\infty)$ is a continuous function, and assume that $V(t, u)$ satisfies the following assumption:

$\left(V_{0}\right) V(t, u)$ is measurable in $t$ for each $u \in \mathbb{R}^{N}$ and continuously differentiable in $u$ for a.e. $t \in[0, T]$, and there exist $a \in C\left(\mathbb{R}^{+}, \mathbb{R}^{+}\right), b \in L^{1}\left([0, T] ; \mathbb{R}^{+}\right)$such that

$$
|V(t, u)|+|\nabla V(t, u)| \leq a(|u|) b(t)
$$

for all $u \in \mathbb{R}^{N}$ and a.e. $t \in[0, T]$.

Throughout the paper, we assume that $p(t)$ appearing in problem (1.3) satisfies

(P) $p(t) \in C\left([0, T], \mathbb{R}^{+}\right), p(t)=p(t+T)$ and $p(t)$ fulfills the following hypothesis:

$$
1<p^{-}:=\min _{0 \leq t \leq T} p(t) \leq p^{+}:=\max _{0 \leq t \leq T} p(t)<+\infty
$$

Comparing with systems (1.1) or (1.2), one typical feature of the equation in problem (1.3) is the nonlocality. Since the presence of an integral term $M\left(\int_{0}^{T} \frac{|\dot{u}(t)|^{p(t)}}{p(t)} d t\right)$, thus the equation in problem (1.3) is no longer a pointwise identity. From the physical point of view, the nonlocal coefficient $M\left(\int_{0}^{T} \frac{\mid \dot{u}(t) p^{p(t)}}{p(t)} d t\right)$ is a function depending on the average of the kinetic energy. Alternatively, a parabolic version of problem (1.3) can model a spreading process of a particular species within the domain, where $u$ is its population density; see [16]. During the past decade, much interest has grown on nonlocal problems with various boundary data. In the very recent paper [17], the authors studied an elliptic boundary value problem with degenerate nonlocal term, and they proved a multiplicity result of positive solutions for the problem, where the number of solutions doubles the number of zeros of the degenerate term. In [18], the authors investigated a class of Kirchhoff problem in a bounded domain, where the nonlocal coefficient to vanish in many different points, and the existence of multiple solutions is also established by using a priori estimates, variational methods and truncation techniques. 
It is worth mentioning that there are some papers concerning related equations or abstract spaces with variable exponents. In the monograph [19], Radulescu and Repovs provided a thorough introduction to the theory of nonlinear partial differential equations (PDEs) with a variable exponent. In [20], the authors considered a class of double phase variational integrals driven by non-homogeneous potentials, and the analysis developed in this paper extends the abstract framework corresponding to some standard differential operator with variable exponent. In [21], Ho and Sim obtained the existence of solutions of weighted elliptic equations containing a convection term with variable exponents. Liu and Zhao in [22] introduced the definition of abstract Hardy spaces with variable exponents. In [23], the authors proved continuity of the flow and weak upper semicontinuity of a family of global attractors for reaction-diffusion equations with spatially variable exponents. In recent years, some scholars have investigated nonlocal $p(x)$-Laplacian equations with Dirichlet or Neumann boundary condition; see [24-27]. However, as far as we known, there are few papers discussing periodic solutions for nonlocal problems (1.3). For instance, by means of a variational analysis and the theory of variable exponent spaces, the author in [28] obtained the existence and multiplicity of periodic solutions for systems (1.3) with $p^{-}$-sublinear nonlinear term.

For $p(t)$-Laplacian systems, the well-known Ambrosetti-Rabinowitz type superlinearity condition (AR-condition, for short) is the following: there exist $R>0$ and $\mu>p^{+}$such that

$$
0<\mu V(t, u) \leq(\nabla V(t, u), u)
$$

for $|u| \geq R$ and $t \in[0, T]$. This kinds of technical condition implies that $\nabla V(t, u)$ grows at a $p^{+}$-superlinear rate as $|u| \rightarrow+\infty$. The main role of AR-condition is to ensure the compactness required by minimax arguments, and without the AR-condition the situation is more complicated. However, there are many functions which are superlinear at infinity, but do not satisfy the AR-condition, and these functions have attracted much interest in recent years, for example; see [29-33].

In this paper, under no AR-condition, we study the existence of nontrivial periodic solutions for problem (1.3) with $p^{+}$-superlinear nonlinear terms at infinity.

In the sequel, we will assume that $M(s):[0,+\infty) \rightarrow(0,+\infty)$ is continuous and

$\left(M_{0}\right)$ there exists a constant $m_{0}>0$ such that $M(s) \geq m_{0}$, for all $s \in[0,+\infty)$;

$\left(M_{1}\right)$ there exists a constant $\eta \geq 1$ such that

$$
\widehat{M}(s):=\int_{0}^{s} M(\sigma) d \sigma \geq \frac{1}{\eta} M(s) s,
$$

for all $s \in[0,+\infty)$.

Now, we suppose that $V:[0, T] \times \mathbb{R}^{N} \rightarrow \mathbb{R}$ satisfies the following assumption:

$\left(V_{1}\right) \quad V(t, u) \geq 0$, for all $u \in \mathbb{R}^{N}$ and a.e. $t \in[0, T]$;

$\left(V_{2}\right) \lim \sup _{|u| \rightarrow 0} \frac{|V(t, u)|}{|u|^{p^{+}}} \leq \frac{m_{0}}{2 p^{+} T C_{0}^{p^{+}}}$, uniformly for a.e. $t \in[0, T]$;

$\left(V_{3}\right)$ there exists a decreasing function $h \in C\left(\mathbb{R}^{+}, \mathbb{R}^{+}\right), r>0$, such that

$$
\left(\eta p^{+}+h(|u|)\right) V(t, u) \leq(\nabla V(t, u), u)
$$

for a.e. $t \in[0, T]$ and $u \in \mathbb{R}^{N}$ with $|u| \geq r$, where $h$ satisfies the following properties: 
$\left(h_{1}\right) \lim _{|u| \rightarrow+\infty} u h(|u|)=+\infty, \forall u \in \mathbb{R}^{+}$

$\left(h_{2}\right) \lim _{|u| \rightarrow+\infty} H(|u|)=+\infty$, where $H(|u|)=\exp \left(\int_{r}^{|u|} \frac{h(\sigma)}{\sigma} d \sigma\right)$.

$\left(V_{4}\right) \liminf _{|u| \rightarrow+\infty} \frac{V(t, u)}{|u|^{\eta p^{+}} H(|u|)} \geq \kappa>0$, uniformly for a.e. $t \in[0, T]$.

Our main result is the following theorem.

Theorem 1.1 Assume that conditions $(P),\left(M_{0}\right),\left(M_{1}\right)$ and $\left(V_{0}\right)-\left(V_{4}\right)$ are satisfied. Then problem (1.3) has at least one nontrivial $T$-periodic solution.

Remark 1.1 Take the nonlocal coefficient

$$
M(s)=1+\frac{\cos s}{1+s^{2}},
$$

then $M$ satisfies $\left(M_{0}\right)$ and $\left(M_{1}\right)$ with $m_{0}=1$ and $\eta=1$, let

$$
V(t, u)=f(t)|u|^{p^{+}} \ln (1+|u|), \quad h(|u|)=\frac{|u|}{2(1+|u|) \ln (1+|u|)},
$$

where $f(t) \in L^{1}\left([0, T] ; \mathbb{R}^{+}\right)$with $\inf _{t \in[0, T]} f(t)>0$. Hence, we obtain

$$
(\nabla V(t, u), u)=f(t)\left[p^{+}+\frac{|u|}{(1+|u|) \ln (1+|u|)}\right]|u|^{p^{+}} \ln (1+|u|) .
$$

Then all the conditions of Theorem 1.1 hold, and $V$ is not covered by results in [7-26].

Next, we obtain the existence of periodic solutions for problem (1.3) with $p^{-}$-subquadratic potential. Now, we state the assumptions on function $M$ and $V$ :

$\left(M_{2}\right)$

$$
\widehat{M}(s):=\int_{0}^{s} M(\sigma) d \sigma \leq M(s) s,
$$

for all $s \in[0,+\infty)$.

$\left(V_{5}\right)$ There exists a function $\theta$ with $0<\frac{1}{\theta(|u|)}<p^{-}, L>0$ such that

$$
(\nabla V(t, u), u) \leq\left(p^{-}-\frac{1}{\theta(|u|)}\right) V(t, u)
$$

for all $u \in \mathbb{R}^{N}$ with $|u| \geq L$ and a.e. $t \in[0, T]$, where $\theta$ satisfies the following properties:

$\left(\theta_{1}\right) \quad \theta \in C\left(\mathbb{R}^{+}, \mathbb{R}^{+}\right)$

$\left(\theta_{2}\right) \int_{L}^{\xi} \frac{1}{s \theta(s)} d s \rightarrow+\infty$ as $\xi \rightarrow+\infty$.

$\left(V_{6}\right) V(t, u) \geq 0$ as $|u| \rightarrow+\infty$ uniformly for a.e. $t \in[0, T]$.

$\left(V_{7}\right) \int_{0}^{T} \frac{V(t, u)}{\theta(|u|)} d t \rightarrow+\infty$ as $|u| \rightarrow+\infty$.

Theorem 1.2 Assume that conditions $(P),\left(M_{0}\right),\left(M_{2}\right),\left(V_{0}\right),\left(V_{5}\right),\left(V_{6}\right)$ and $\left(V_{7}\right)$ are satisfied. Then problem (1.3) has at least one T-periodic solution.

Remark 1.2 When $M(s) \equiv 1, p(t) \equiv 2$, condition $\left(V_{5}\right)$ was introduced in Wang and Xiao [27], which is an extension of the usual subquadratic growth condition. Theorem 1.2 generalizes Theorem 1.1 of Wang and Xiao [34] to the nonlocal and variable exponent space setting. 
Remark 1.3 When $M(s) \equiv 1$, Wang and Yuan [9] prove that problem (1.1) admits a $T$ periodic solution when the function $V(t, u)$ is $p^{-}$-subquadratic in Rabinowitz's sense, that is, there exists $L>0,0<\mu<p^{-}$such that

$$
(\nabla V(t, u), u) \leq \mu V(t, u)
$$

for all $|u| \geq L$ and a.e. $t \in[0, T]$.

Define $\inf _{|u| \geq L} \frac{1}{\theta(|u|)}:=\mathcal{K}$, where $\mathcal{K}$ is a constant. If $\mathcal{K}>0$, then condition $\left(V_{5}\right)$ and (1.5) are equivalent. However, condition $\left(V_{5}\right)$ is weaker than (1.5) when $\mathcal{K}=0$.

Remark 1.4 The following example is presented for Theorem 1.2. For $s \in[0,+\infty)$, let

$$
M(s)=A+B s^{m}
$$

where $A, B$ and $m$ are positive constants. Then the nonlocal coefficient $M$ satisfies $\left(M_{0}\right)$ and $\left(M_{2}\right)$ with $m_{0}=A$. Assume that $p(t) \equiv \sin \frac{2 \pi t}{T}+5$, then $p^{-}=4$, let

$$
V(t, u)=g(t) \frac{4+|u|^{4}}{\ln \left(1+|u|^{4}\right)}
$$

where

$$
g(t)= \begin{cases}\sin \frac{2 \pi t}{T}, & t \in[0, T / 2] \\ 0, & t \in[T / 2, T]\end{cases}
$$

Setting $\theta(|u|)=\ln \left(1+|u|^{4}\right)$, then $V(t, u)$ satisfies $\left(V_{5}\right)$ but not (1.5).

\section{Preliminaries}

In this section, we recall some properties of the variable exponent Sobolev space.

Definition $2.1([8])$ Let $p(t) \in C\left([0, T], \mathbb{R}^{+}\right)$, and $p(t)$ satisfies $(P)$. Define

$$
L^{p(t)}\left([0, T], \mathbb{R}^{N}\right)=\left\{\left.u \in L^{1}\left([0, T], \mathbb{R}^{N}\right)\left|\int_{0}^{T}\right| u(t)\right|^{p(t)} d t<+\infty\right\},
$$

with the norm

$$
|u|_{p(t)}=\inf \left\{\lambda>\left.0\left|\int_{0}^{T}\right| \frac{u(t)}{\lambda}\right|^{p(t)} d t \leq 1\right\}
$$

Definition 2.2 ([8]) Define

$$
C_{T}^{\infty}=C_{T}^{\infty}\left(\mathbb{R}, \mathbb{R}^{N}\right)=\left\{u \in C^{\infty}\left(\mathbb{R}, \mathbb{R}^{N}\right) \mid u \text { is } T \text {-periodic }\right\} .
$$

Let $u \in L^{1}\left([0, T], \mathbb{R}^{N}\right)$ and $v \in L^{1}\left([0, T], \mathbb{R}^{N}\right)$. If

$$
\int_{0}^{T} v \phi d t=-\int_{0}^{T} u \phi^{\prime} d t, \quad \forall \phi \in C_{T}^{\infty}
$$

then $v$ is called the $T$-weak derivative of $u$ and is denoted by $\dot{u}$. 
Definition 2.3 ([8]) Define

$$
W_{T}^{1, p(t)}=\left\{u \in L^{p(t)}\left([0, T], \mathbb{R}^{N}\right) \mid \dot{u} \in L^{p(t)}\left([0, T], \mathbb{R}^{N}\right), u(0)=u(T)\right\} .
$$

When $p^{-}>1, W_{T}^{1, p(t)}$ is reflexive Banach space, with the norm

$$
\|u\|_{W_{T}^{1, p(t)}}=|u|_{p(t)}+|\dot{u}|_{p(t)}
$$

For $u \in W_{T}^{1, p(t)}$, we write

$$
\|u\|=|\bar{u}|+|\dot{u}|_{p(t)}
$$

then $\|\cdot\|$ is an equivalent norm on $W_{T}^{1, p(t)}$, where $\bar{u}=\frac{1}{T} \int_{0}^{T} u(t) d t$.

Lemma 2.1 ([8]) If we denote

$$
\rho(u)=\int_{0}^{T}|u(t)|^{p(t)} d t, \quad \forall u \in L^{p(t)}\left([0, T], \mathbb{R}^{N}\right),
$$

then

(i) $|u|_{p(t)}<1(=1 ;>1) \Leftrightarrow \rho(u)<1(=1 ;>1)$;

(ii) $|u|_{p(t)}>1 \Rightarrow|u|_{p(t)}^{p^{-}} \leq \rho(u) \leq|u|_{p(t)}^{p^{+}}$;

(iii) $|u|_{p(t)}<1 \Rightarrow|u|_{p(t)}^{p^{+}} \leq \rho(u) \leq|u|_{p(t)}^{p^{-}}$.

Lemma 2.2 ([7]) There is a continuous embedding $W_{T}^{1, p(t)} \hookrightarrow C\left([0, T], \mathbb{R}^{N}\right)$, when $p^{-}>1$, the embedding is compact. Then there exists $C_{0}>0$, such that

$$
\|u\|_{\infty}:=\max _{0 \leq t \leq T}|u(t)| \leq C_{0}\|u\|, \quad \forall u \in W_{T}^{1, p(t)}
$$

Lemma 2.3 ([9]) Let

$$
\Phi(u)=\int_{0}^{T}\left(|\dot{u}(t)|^{p(t)}+|u(t)|^{p(t)}\right) d t, \quad \forall u \in W_{T}^{1, p(t)}
$$

then

(i) $\|u\|<(=;>) 1 \Leftrightarrow \Phi(u)<(=;>) 1$;

(ii) $\|u\|>1 \Rightarrow\|u\|^{p^{-}} \leq \Phi(u) \leq\|u\|^{p^{+}}$;

(iii) $\|u\|<1 \Rightarrow\|u\|^{p^{+}} \leq \Phi(u) \leq\|u\|^{p^{-}}$.

Lemma $2.4([9]) J^{\prime}$ is a bounded linear functional and a mapping of $\left(S_{+}\right)$on $W_{T}^{1, p(t)}$, that is, if $u_{n} \rightarrow u$ weakly in $W_{T}^{1, p(t)}$ and $\limsup _{n \rightarrow \infty}\left(J^{\prime}\left(u_{n}\right)-J^{\prime}(u), u_{n}-u\right) \leq 0$, then $\left\{u_{n}\right\}$ has a convergent subsequence, where $J^{\prime}$ is given by $\left\langle J^{\prime}(u), v\right\rangle=\int_{0}^{T}\left(|\dot{u}(t)|^{p(t)-2} \dot{u}(t), \dot{v}(t)\right) d t$.

For $u \in W_{T}^{1, p(t)}$, we can write $u(t)=\bar{u}+\tilde{u}(t)$, where $\bar{u}=\frac{1}{T} \int_{0}^{T} u(t) d t$. Let

$$
\widetilde{W}_{T}^{1, p(t)}=\left\{u \in W_{T}^{1, p(t)} \mid \bar{u}=0\right\} .
$$

It is easy to know $\widetilde{W}_{T}^{1, p(t)}$ is a subset of $W_{T}^{1, p(t)}$ and $W_{T}^{1, p(t)}=\widetilde{W}_{T}^{1, p(t)} \oplus \mathbb{R}^{N}$.

Applying Lemma 2.1, from (2.1), it is easy to prove that 
Lemma 2.5 For all $\tilde{u} \in \widetilde{W}_{T}^{1, p(t)}$, we have

(i) $\|\widetilde{u}\|>1 \Rightarrow\|\widetilde{u}\|^{p^{-}} \leq \int_{0}^{T}|\dot{u}(t)|^{p(t)} d t \leq\|\widetilde{u}\|^{p^{+}}$;

(ii) $\|\widetilde{u}\|<1 \Rightarrow\|\widetilde{u}\|^{p^{+}} \leq \int_{0}^{T}|\dot{u}(t)|^{p(t)} d t \leq\|\widetilde{u}\|^{p^{-}}$;

(iii) $\|\tilde{u}\|=1 \Rightarrow \int_{0}^{T}|\dot{u}(t)|^{p(t)} d t=1$.

Lemma 2.6 For all $\tilde{u} \in \widetilde{W}_{T}^{1, p(t)}$, we have

$$
\|\widetilde{u}\|^{p^{-}} \leq \int_{0}^{T}|\dot{u}(t)|^{p(t)} d t+1 .
$$

\section{Proof of Theorem 1.1}

As usual, a weak solution of problem (1.3) is a function $u \in W_{T}^{1, p(t)}$ such that

$$
M\left(\int_{0}^{T} \frac{|\dot{u}(t)|^{p(t)}}{p(t)} d t\right) \int_{0}^{T}\left(|\dot{u}(t)|^{p(t)-2} \dot{u}(t), \dot{v}(t)\right) d t=\int_{0}^{T}(\nabla V(t, u(t)), v(t)) d t
$$

holds for any $v \in W_{T}^{1, p(t)}$.

The Euler-Lagrange functional associated to problem(1.3) given by

$$
I(u)=\widehat{M}\left(\int_{0}^{T} \frac{|\dot{u}(t)|^{p(t)}}{p(t)} d t\right)-\int_{0}^{T} V(t, u(t)) d t, \quad \forall u \in W_{T}^{1, p(t)},
$$

where

$$
\widehat{M}(s)=\int_{0}^{s} M(\sigma) d \sigma .
$$

Then $I \in C^{1}\left(W_{T}^{1, p(t)}, \mathbb{R}\right)$ whose Gateaux derivative is

$$
\begin{aligned}
\left\langle I^{\prime}(u), v\right\rangle= & M\left(\int_{0}^{T} \frac{|\dot{u}(t)|^{p(t)}}{p(t)} d t\right) \int_{0}^{T}\left(|\dot{u}(t)|^{p(t)-2} \dot{u}(t), \dot{v}(t)\right) d t \\
& -\int_{0}^{T}(\nabla V(t, u(t)), v(t)) d t,
\end{aligned}
$$

for all $u, v \in W_{T}^{1, p(t)}$. So we know that $T$-periodic weak solutions of problem (1.3) correspond to the critical points of the functional $I$.

A basic tool in this paper is the following abstract local linking theorem.

Theorem 3.1 ([35]) Let $X$ be a Banach space such that $X=Y \oplus W$ with $\operatorname{dim} Y<+\infty$. Assume that $I \in C^{1}(X, \mathbb{R})$ satisfies the following conditions:

$\left(I_{1}\right)$ I satisfies the $(C)$ condition, that is, sequence $\left\{u_{n}\right\} \subset X$ such that $\left\{I\left(u_{n}\right)\right\}$ is bounded and $\left\|I^{\prime}\left(u_{n}\right)\right\|\left(1+\left\|u_{n}\right\|\right) \rightarrow 0$ as $n \rightarrow \infty$ has a convergent sequence.

$\left(I_{2}\right)$ I has a local linking at zero, that is, there exists a positive constant $\delta$ such that

$$
I(u) \leq 0, \quad \forall u \in Y,\|u\| \leq \delta ;
$$

and

$$
I(u) \geq 0, \quad \forall u \in W,\|u\| \leq \delta .
$$


$\left(I_{3}\right)$ I maps bounded sets into bounded sets.

$\left(I_{4}\right)$ for every finite-dimensional subspace $\widetilde{E} \subseteq W$, we have

$$
I(u) \rightarrow-\infty \quad \text { as }\|u\| \rightarrow+\infty \text { and } u \in Y \oplus \widetilde{E} .
$$

Then I admits at least one nontrivial critical point.

Remark 3.1 The critical point theorem was originally due to Li and Willem [36]. Subsequently, Gasiński and Papageorgiou reformulate this result in the special case given in [35, Theorem 2.1].

In the sequel, we will denote various positive constants as $D_{i}(i=1,2,3, \ldots)$.

Lemma 3.1 Suppose $(P),\left(M_{0}\right),\left(M_{1}\right),\left(V_{0}\right),\left(V_{1}\right),\left(V_{3}\right)$ and $\left(V_{4}\right)$ hold. Then I satisfies the (C) condition, that is, sequence $\left\{u_{n}\right\} \subset W_{T}^{1, p(t)}$ such that $\left\{I\left(u_{n}\right)\right\}$ is bounded and $\left\|I^{\prime}\left(u_{n}\right)\right\|(1+$ $\left.\left\|u_{n}\right\|\right) \rightarrow 0$ as $n \rightarrow+\infty$ has a convergent sequence.

Proof Let $\left\{u_{n}\right\} \subset W_{T}^{1, p(t)}$, such that $\left\{I\left(u_{n}\right)\right\}$ is bounded and $\left(1+\left\|u_{n}\right\|\right)\left\|I^{\prime}\left(u_{n}\right)\right\| \rightarrow 0$ as $n \rightarrow$ $\infty$. There then exists a constant $D_{1}>0$ such that

$$
\left|I\left(u_{n}\right)\right| \leq D_{1}, \quad\left(1+\left\|u_{n}\right\|\right)\left\|I^{\prime}\left(u_{n}\right)\right\| \leq D_{1},
$$

for all $n$. We claim that the sequence $\left\{u_{n}\right\}$ is bounded in $W_{T}^{1, p(t)}$. Suppose that is not the case, passing to a subsequence if necessary, we may assume that

$$
\lim _{n \rightarrow+\infty}\left\|u_{n}\right\|=+\infty
$$

Note that $p^{+}:=\max _{0 \leq t \leq T} p(t)$, using hypothesis $\left(M_{1}\right)$, one has

$$
\begin{aligned}
& \widehat{M}\left(\int_{0}^{T} \frac{\left|\dot{u}_{n}(t)\right|^{p(t)}}{p(t)} d t\right) \\
& \quad \geq \frac{1}{\eta} M\left(\int_{0}^{T} \frac{\left|\dot{u}_{n}(t)\right|^{p(t)}}{p(t)} d t\right) \int_{0}^{T} \frac{\left|\dot{u}_{n}(t)\right|^{p(t)}}{p(t)} d t \\
& \quad \geq \frac{1}{\eta p^{+}} M\left(\int_{0}^{T} \frac{\left|\dot{u}_{n}(t)\right|^{p(t)}}{p(t)} d t\right) \int_{0}^{T}\left|\dot{u}_{n}(t)\right|^{p(t)} d t .
\end{aligned}
$$

Set

$$
\begin{aligned}
& K:=\inf _{|u|>r} h(|u|)>0, \\
& E_{1}:=\{t \in[0, T]|| u \mid>r\},
\end{aligned}
$$

and

$$
E_{2}:=\{t \in[0, T]|| u \mid \leq r\}
$$


It follows from $\left(V_{0}\right),\left(V_{1}\right)$ and $\left(V_{3}\right)$ that

$$
\begin{aligned}
\int_{0}^{T} & {\left[(\nabla V(t, u), u)-\left(\eta p^{+}+K\right) V(t, u)\right] d t } \\
= & \int_{E_{1}}\left[(\nabla V(t, u), u)-\left(\eta p^{+}+K\right) V(t, u)\right] d t \\
& +\int_{E_{2}}\left[(\nabla V(t, u), u)-\left(\eta p^{+}+K\right) V(t, u)\right] d t \\
\geq & \int_{E_{1}}\left[(\nabla V(t, u), u)-\left(\eta p^{+}+h(|u|)\right) V(t, u)\right] d t \\
& +\int_{E_{2}}\left|(\nabla V(t, u), u)-\left(\eta p^{+}+K\right) V(t, u)\right| d t \\
\geq & -\left(r+\eta p^{+}+K\right) \max _{|u| \leq r} a(|u|) \int_{0}^{T} b(t) d t .
\end{aligned}
$$

By (3.1), (3.3), (3.4) and $\left(M_{0}\right)$, we have

$$
\begin{aligned}
D_{2} \geq & I\left(u_{n}\right)+\frac{1}{\eta p^{+}+K}\left(1+\left\|u_{n}\right\|\right)\left\|I^{\prime}\left(u_{n}\right)\right\| \\
\geq & I\left(u_{n}\right)-\frac{1}{\eta p^{+}+K}\left\langle I^{\prime}\left(u_{n}\right), u_{n}\right\rangle \\
= & \widehat{M}\left(\int_{0}^{T} \frac{\left|\dot{u}_{n}(t)\right|^{p(t)}}{p(t)} d t\right)-\frac{M\left(\int_{0}^{T} \frac{\mid \dot{u}_{n}(t) p(t)}{p(t)} d t\right)}{\eta p^{+}+K} \int_{0}^{T}\left|\dot{u}_{n}(t)\right|^{p(t)} d t \\
& +\int_{0}^{T}\left[\frac{1}{\eta p^{+}+K}\left(\nabla V\left(t, u_{n}\right), u_{n}\right)-V\left(t, u_{n}\right)\right] d t \\
\geq & \left(\frac{1}{\eta p^{+}}-\frac{1}{\eta p^{+}+K}\right) M\left(\int_{0}^{T} \frac{\left|\dot{u}_{n}(t)\right|^{p(t)}}{p(t)} d t\right) \int_{0}^{T}\left|\dot{u}_{n}(t)\right|^{p(t)} d t \\
& -\frac{r+\eta p^{+}+K}{\eta p^{+}+K} \max _{|u| \leq r} a(|u|) \int_{0}^{T} b(t) d t \\
\geq & \left(\frac{1}{\eta p^{+}}-\frac{1}{\eta p^{+}+K}\right) m_{0} \int_{0}^{T}\left|\dot{u}_{n}(t)\right|^{p(t)} d t-\frac{r+\eta p^{+}+K}{\eta p^{+}+K} \max _{|u| \leq r} a(|u|) \int_{0}^{T} b(t) d t,
\end{aligned}
$$

which implies that

$$
\int_{0}^{T}\left|\dot{u}_{n}(t)\right|^{p(t)} d t \leq D_{3} .
$$

Combining (2.3) with (3.5), we have

$$
\left\|\tilde{u}_{n}\right\|^{p^{-}} \leq D_{3}+1
$$

For $\forall u \in W_{T}^{1, p(t)}$, let $\bar{u}=\frac{1}{T} \int_{0}^{T} u(t) d t \in \mathbb{R}^{N}, \widetilde{u}(t)=u(t)-\bar{u}$. Set

$$
\omega_{n}=\frac{u_{n}}{\left\|u_{n}\right\|}=\frac{\bar{u}_{n}}{\left\|u_{n}\right\|}+\frac{\tilde{u}_{n}}{\left\|u_{n}\right\|}=\bar{\omega}_{n}+\widetilde{\omega}_{n},
$$


then $\left\{\omega_{n}\right\}$ is bounded in $W_{T}^{1, p(t)}$ and $\left\|\omega_{n}\right\|=1$. Hence, up to a subsequence, we get

$$
\omega_{n} \rightarrow \omega \quad \text { weakly in } W_{T}^{1, p(t)}
$$

and

$$
\omega_{n} \rightarrow \omega \text { strongly in } C\left([0, T] ; \mathbb{R}^{N}\right) .
$$

Dividing both side of (3.6) by $\left\|u_{n}\right\|^{p^{-}}$, from (3.2) and (3.6), we find that

$$
\lim _{n \rightarrow+\infty}\left\|\widetilde{\omega}_{n}\right\|=0
$$

So we deduce that $\omega=\bar{\omega} \in \mathbb{R}^{N}$ and $\omega \neq 0$. Then, by (3.2), we infer that

$$
\lim _{n \rightarrow+\infty} u_{n}(t)=+\infty
$$

uniformly for a.e. $t \in[0, T]$. By $\left(V_{1}\right),\left(V_{4}\right),\left(h_{2}\right),(3.8)$ and Fatou's lemma, we get

$$
\begin{aligned}
& \liminf _{n \rightarrow+\infty} \int_{0}^{T} \frac{V\left(t, u_{n}(t)\right)}{\left\|u_{n}\right\| \eta p^{+}} d t \\
& \quad \geq \int_{0}^{T} \liminf _{n \rightarrow+\infty} \frac{V\left(t, u_{n}(t)\right)}{\left\|u_{n}\right\|^{\eta p^{+}}} d t \\
& \quad=\int_{0}^{T} \liminf _{n \rightarrow+\infty} \frac{V\left(t, u_{n}(t)\right)}{\left|u_{n}(t)\right|^{\eta p^{+}} H\left(\left|u_{n}(t)\right|\right)} \mid \omega_{n}\left(\left.t\right|^{\eta p^{+}} H\left(\left|u_{n}(t)\right|\right) d t\right. \\
& \quad \geq \int_{0}^{T} \liminf _{n \rightarrow+\infty} \frac{V\left(t, u_{n}(t)\right)}{\left|u_{n}(t)\right|^{\eta p^{+}} H\left(\left|u_{n}(t)\right|\right)}|\omega|^{\eta p^{+}} H\left(\left|u_{n}(t)\right|\right) d t \\
& \quad=+\infty
\end{aligned}
$$

Set $s_{1}>0$, by condition $\left(M_{1}\right)$, we obtain

$$
\frac{M(s)}{\widehat{M}(s)} \leq \frac{\eta}{s},
$$

for every $s \in\left[s_{1},+\infty\right)$, where $\eta \geq 1$. Integrating the above inequality we obtain

$$
\begin{aligned}
\ln \frac{\widehat{M}(s)}{\widehat{M}\left(s_{1}\right)} & =\int_{s_{1}}^{s} \frac{M(\sigma)}{\widehat{M}(\sigma)} d \sigma \\
& \leq \int_{s_{1}}^{s} \frac{\eta}{\sigma} d \sigma \\
& =\ln \left(\frac{s}{s_{1}}\right)^{\eta},
\end{aligned}
$$

for every $s \in\left[s_{1},+\infty\right)$, where $\widehat{M}(s)=\int_{0}^{s} M(\sigma) d \sigma$. Therefore,

$$
\widehat{M}(s) \leq \frac{\widehat{M}\left(s_{1}\right)}{s_{1}^{\eta}} s^{\eta},
$$


for every $s \in\left[s_{1},+\infty\right)$. Thus there exist constants $m_{1}>0$ and $m_{2}>0$, such that

$$
\widehat{M}(s) \leq m_{1} s^{\eta}+m_{2},
$$

for all $s>0$, where $m_{1}:=\frac{\widehat{M}\left(s_{1}\right)}{s_{1}^{\eta}}$ and $m_{2}:=\max _{s \in\left[0, s_{1}\right]} \widehat{M}(s)$.

According to (3.5) and (3.10), it suffices to show that

$$
\begin{aligned}
I\left(u_{n}\right)+\int_{0}^{T} V\left(t, u_{n}(t)\right) d t & =\widehat{M}\left(\int_{0}^{T} \frac{\left|\dot{u}_{n}(t)\right|^{p(t)}}{p(t)} d t\right) \\
& \leq m_{1}\left(\int_{0}^{T} \frac{\left|\dot{u}_{n}(t)\right|^{p(t)}}{p(t)} d t\right)^{\eta}+m_{2} \\
& \leq m_{1}\left(\frac{1}{p^{-}}\right)^{\eta}\left(\int_{0}^{T}\left|\dot{u}_{n}(t)\right|^{p(t)} d t\right)^{\eta}+m_{2} \\
& \leq D_{4} .
\end{aligned}
$$

It follows from (3.1), (3.2), (3.11) and $\left(V_{1}\right)$ that

$$
\limsup _{n \rightarrow+\infty} \int_{0}^{T} \frac{V\left(t, u_{n}(t)\right)}{\left\|u_{n}\right\|^{\eta p^{+}}} d t \leq 0
$$

which contradicts (3.9). Hence, $\left\{u_{n}\right\}$ is bounded in $W_{T}^{1, p(t)}$.

Notice that the space $W_{T}^{1, p(t)}$ is reflexive, the sequence $\left\{u_{n}\right\} \subset W_{T}^{1, p(t)}$ has a subsequence, also denoted by $\left\{u_{n}\right\}$, such that

$$
u_{n} \rightarrow u \quad \text { weakly in } W_{T}^{1, p(t)}
$$

and

$$
u_{n} \rightarrow u \quad \text { strongly in } C\left([0, T] ; \mathbb{R}^{N}\right) \text {, }
$$

and $\left\|u_{n}\right\|_{\infty} \leq D_{5}$ by (2.2), where $D_{5}$ is a positive constant. So we have

$$
\begin{aligned}
\left|\int_{0}^{T}\left(\nabla V\left(t, u_{n}(t)\right), u_{n}(t)-u(t)\right) d t\right| & \leq a_{0} T\left\|u_{n}-u\right\|_{\infty} \int_{0}^{T} b(t) d t \\
& \rightarrow 0 \text { as } n \rightarrow+\infty,
\end{aligned}
$$

where $a_{0}=\max _{0 \leq s \leq D_{5}} a(s)$. By the assumption $\left(1+\left\|u_{n}\right\|\right)\left\|I^{\prime}\left(u_{n}\right)\right\| \rightarrow 0$ as $n \rightarrow+\infty$, we have

$$
\left\langle I^{\prime}\left(u_{n}\right), u_{n}-u\right\rangle \rightarrow 0 \quad \text { as } n \rightarrow+\infty
$$

Thus

$$
M\left(\int_{0}^{T}\left|\dot{u}_{n}(t)\right|^{p(t)} d t\right) \int_{0}^{T}\left(\left|\dot{u}_{n}(t)\right|^{p(t)-2} \dot{u}_{n}(t), \dot{u}_{n}(t)-\dot{u}(t)\right) d t \rightarrow 0 \quad \text { as } n \rightarrow+\infty .
$$


Let $J^{\prime}: W_{T}^{1, p(t)} \rightarrow\left(W_{T}^{1, p(t)}\right)^{*}$ defined by

$$
\left\langle J^{\prime}(u), v\right\rangle=\int_{0}^{T}\left(|\dot{u}(t)|^{p(t)-2} \dot{u}(t), \dot{v}(t)\right) d t .
$$

Then, by $\left(M_{0}\right)$, we get $\lim _{n \rightarrow+\infty}\left(J^{\prime}\left(u_{n}\right), u_{n}-u\right)=0$. Furthermore, since $J^{\prime}(u)$ is bounded linear function, we get $\lim _{n \rightarrow+\infty}\left(J^{\prime}(u), u_{n}-u\right)=0$. Thus

$$
\lim _{n \rightarrow+\infty}\left(J^{\prime}\left(u_{n}\right)-J^{\prime}(u), u_{n}-u\right)=0 .
$$

Hence, using the $\left(S_{+}\right)$property (see Lemma 2.4), $J^{\prime}$ is a bounded linear functional and a mapping of $\left(S_{+}\right)$on $W_{T}^{1, p(t)}$, it follows that $\left\{u_{n}\right\}$ has a convergent subsequence. So we see that $\left\{u_{n}\right\}$ admits a convergent sequence. Thus $I$ satisfies condition $(C)$.

Now we prove our main result Theorem 1.1.

Proof of Theorem 1.1 By Lemma 3.1 we have $I$ satisfies $\left(I_{1}\right)$ of Theorem 3.1, so it suffices to prove $\left(I_{2}\right),\left(I_{3}\right)$ and $\left(I_{4}\right)$ of Theorem 3.1.

Claim 1 Let $W_{T}^{1, p(t)}=\widetilde{W}_{T}^{1, p(t)} \oplus \mathbb{R}^{N}$, we claim that $I$ has a local linking at zero.

On the one hand, in view of condition $\left(V_{2}\right)$, there exist two constants $\rho$ and $\varepsilon$ such that

$$
0<\rho<\varepsilon<\min \left\{C_{0}, \frac{m_{0}}{2 p^{+} T C_{0}^{p^{+}}}\right\}
$$

where $C_{0}$ is the same as in (2.2), and

$$
|V(t, u)| \leq\left(\frac{m_{0}}{2 p^{+} T C_{0}^{p^{+}}}+\varepsilon\right)|u|^{p^{+}}
$$

for a.e. $t \in[0, T]$ and $|u| \leq \rho$. Let $\delta:=\rho / C_{0}$, then $\delta<1$. If $\|u\| \leq \delta$, we have

$$
|u(t)| \leq\|u\|_{\infty} \leq C_{0}\|u\| \leq \rho .
$$

By $\left(M_{0}\right)$, (3.13), (3.14) and Lemma 2.5 , for $u \in \widetilde{W}_{T}^{1, p(t)}$ with $\|u\| \leq \delta$, one has

$$
\begin{aligned}
I(u) & =\widehat{M}\left(\int_{0}^{T} \frac{|\dot{u}(t)|^{p(t)}}{p(t)} d t\right)-\int_{0}^{T} V(t, u(t)) d t \\
& =\int_{0}^{\int_{0}^{T} \frac{\mid \dot{|i(t)| p(t)}}{p(t)} d t} M(s) d s-\int_{0}^{T} V(t, u(t)) d t \\
& \geq m_{0} \int_{0}^{T} \frac{|\dot{u}(t)|^{p(t)}}{p(t)} d t-\left(\frac{m_{0}}{2 p^{+} T C_{0}^{p^{+}}}+\varepsilon\right) \int_{0}^{T}|u(t)|^{p^{+}} d t \\
& \geq \frac{m_{0}}{p^{+}} \int_{0}^{T}|\dot{u}(t)|^{p(t)} d t-\left(\frac{m_{0}}{2 p^{+} T C_{0}^{p^{+}}}+\varepsilon\right) T\|u\|_{\infty}^{p^{+}} \\
& \geq \frac{m_{0}}{p^{+}}\|u\|^{p^{+}}-\left(\frac{m_{0}}{2 p^{+} T C_{0}^{p^{+}}}+\varepsilon\right) T C_{0}^{p^{+}}\|u\|^{p^{+}} \\
& =\left(\frac{m_{0}}{2 p^{+}}-T C_{0}^{p^{+}} \varepsilon\right)\|u\|^{p^{+}} .
\end{aligned}
$$

This implies that $I(u) \geq 0$, for $u \in \widetilde{W}_{T}^{1, p(t)}$ with $\|u\| \leq \delta$. 
On the other hand, by $\left(V_{1}\right)$, for all $y \in \mathbb{R}^{N}$, one has

$$
I(y)=-\int_{0}^{T} V(t, y) d t \leq 0
$$

Hence, $I$ has a local linking at zero.

Claim 2 We claim that $I$ maps bounded sets into bounded sets.

For some positive constant $D_{6}$, assume that $\|u\| \leq D_{6}$, by (3.10), Lemma 2.3 and $\left(V_{0}\right)$, we have

$$
\begin{aligned}
|I(u)|= & \left|\widehat{M}\left(\int_{0}^{T} \frac{|\dot{u}(t)|^{p(t)}}{p(t)} d t\right)-\int_{0}^{T} V(t, u(t)) d t\right| \\
\leq & m_{1}\left(\int_{0}^{T} \frac{|\dot{u}(t)|^{p(t)}}{p(t)} d t\right)^{\eta}+m_{2}+\int_{0}^{T}|V(t, u(t))| d t \\
\leq & m_{1}\left(\frac{1}{p^{-}}\right)^{\eta}\left(\int_{0}^{T}|\dot{u}(t)|^{p(t)} d t+\int_{0}^{T}|u(t)|^{p(t)} d t\right)^{\eta}+m_{2} \\
& +\max _{0 \leq s \leq C_{0} D_{6}} a(s) \int_{0}^{T} b(t) d t \\
\leq & m_{1}\left(\frac{1}{p^{-}} \max \left\{\|u\|^{p^{-}},\|u\|^{p^{+}}\right\}\right)^{\eta}+m_{2} \\
& +\max _{0 \leq s \leq C_{0} D_{6}} a(s) \int_{0}^{T} b(t) d t, \\
\leq & m_{1}\left(\frac{1}{p^{-}} \max \left\{\left(D_{6}\right)^{p^{-}},\left(D_{6}\right)^{p^{+}}\right\}\right)^{\eta}+m_{2} \\
& +\max _{0 \leq s \leq C_{0} D_{6}} a(s) \int_{0}^{T} b(t) d t .
\end{aligned}
$$

Hence, we deduce that $I$ maps bounded sets into bounded sets.

Claim 3 Finally, we claim that

$$
I(u) \rightarrow-\infty \quad \text { as }\|u\| \rightarrow+\infty \text {, for } u \in \mathbb{R}^{N} \oplus \widetilde{E},
$$

where $\widetilde{E} \subseteq \widetilde{W}_{T}^{1, p(t)}$ is a finite-dimensional linear subspace.

From $\left(V_{4}\right)$, there exists a constant $R_{1}>0$, such that

$$
V(t, u) \geq \kappa|u|^{\eta p^{+}} H(|u|)>0,
$$

for $u \in \mathbb{R}^{N}$ with $|u| \geq R_{1}$ and a.e. $t \in[0, T]$. Moreover, by $\left(V_{0}\right)$ and (3.15), we can obtain

$$
\int_{0}^{T} V(t, u) d t \geq \kappa \int_{0}^{T}|u|^{\eta p^{+}} H(|u|) d t-D_{7}
$$

for all $u \in \mathbb{R}^{N}$ and a.e. $t \in[0, T]$, where $D_{7}=\max _{|u| \leq R_{1}} a(|u|) \int_{0}^{T} b(t) d t$.

Note that all norms on $\widetilde{E}$ are equivalent, there exist constants $D_{8}>0$, such that

$$
|u|^{\eta p^{+}} \geq D_{8}\|u\|^{\eta p^{+}}
$$


for all $u \in \widetilde{E}$ and a.e. $t \in[0, T]$. Combining (3.16) with (3.17), we have

$$
\int_{0}^{T} V(t, u) d t \geq \kappa D_{8}\|u\|^{\eta p^{+}} \int_{0}^{T} H(|u|) d t-D_{7}
$$

for all $u \in \widetilde{E}$ and a.e. $t \in[0, T]$.

By (3.8), (3.18) and use Lemma 2.3, choosing $\|u\|>1$, we have

$$
\begin{aligned}
I(u)= & \widehat{M}\left(\int_{0}^{T} \frac{|\dot{u}(t)|^{p(t)}}{p(t)} d t\right)-\int_{0}^{T} V(t, u(t)) d t \\
\leq & m_{1}\left(\int_{0}^{T} \frac{|\dot{u}(t)|^{p(t)}}{p(t)} d t\right)^{\eta}+m_{2}-\int_{0}^{T} V(t, u(t)) d t \\
\leq & m_{1}\left(\frac{1}{p^{-}}\right)^{\eta}\left(\int_{0}^{T}|\dot{u}(t)|^{p(t)} d t+\int_{0}^{T}|u(t)|^{p(t)} d t\right)^{\eta} \\
& -\kappa D_{8}\|u\|^{\eta p^{+}} \int_{0}^{T} H(|u|) d t+D_{9} \\
\leq & {\left[m_{1}\left(\frac{1}{p^{-}}\right)^{\eta}-\kappa D_{8} \int_{0}^{T} H(|u|) d t\right]\|u\|^{\eta p^{+}}+D_{9} . }
\end{aligned}
$$

From (3.17) and $\left(h_{2}\right)$, one has

$$
\int_{0}^{T} H(|u|) d t \rightarrow+\infty \quad \text { as }\|u\| \rightarrow+\infty .
$$

Therefore, we can infer that

$$
I(u) \rightarrow-\infty \quad \text { as }\|u\| \rightarrow+\infty \text {, for } u \in \mathbb{R}^{N} \oplus \widetilde{E} .
$$

Now, Theorem 1.1 is proved by Claims 1-3, Lemma 3.1 and Theorem 3.1. So problem (1.1) has at least one nontrivial $T$-periodic solution.

\section{Proof of Theorem 1.2}

In this section, we will denote various positive constants as $C_{i}(i=0,1,2, \ldots)$.

Lemma 4.1 Assume that assumptions $(P),\left(M_{0}\right),\left(M_{2}\right),\left(V_{0}\right),\left(V_{5}\right)$ and $\left(V_{7}\right)$ hold, then the functional I satisfies the compactness condition $(C)$.

Proof Suppose that $\left\{u_{n}\right\}$ be a Cerami sequence in $W_{T}^{1, p(t)}$, such that $\left\{I\left(u_{n}\right)\right\}$ is bounded and $\left(1+\left\|u_{n}\right\|\right)\left\|I^{\prime}\left(u_{n}\right)\right\| \rightarrow 0$ as $n \rightarrow \infty$. There then exists a constant $C_{1}>0$ such that

$$
\left|I\left(u_{n}\right)\right| \leq C_{1}, \quad\left(1+\left\|u_{n}\right\|\right)\left\|I^{\prime}\left(u_{n}\right)\right\| \leq C_{1}
$$

for all $n \in \mathbb{N}$. By assumption $\left(V_{0}\right)$ and $\left(V_{5}\right)$, one has

$$
-d(t)+(\nabla V(t, u), u) \leq\left(p^{-}-\frac{1}{\theta(|u|)}\right) V(t, u),
$$

for a.e. $t \in[0, T]$ and all $u \in \mathbb{R}^{N}$, where $d(t)=\left(p^{-}+L\right) \max _{|u| \leq L} a(|u|) b(t)$. 
By condition $\left(M_{2}\right)$, we have

$$
M\left(\int_{0}^{T} \frac{\left|\dot{u}_{n}(t)\right|^{p(t)}}{p(t)} d t\right) \int_{0}^{T} \frac{\left|\dot{u}_{n}(t)\right|^{p(t)}}{p(t)} d t \geq \widehat{M}\left(\int_{0}^{T} \frac{\left|\dot{u}_{n}(t)\right|^{p(t)}}{p(t)} d t\right) .
$$

It follows from (4.2) and (4.3) that

$$
\begin{aligned}
C_{2} \geq & \left(1+\left\|u_{n}\right\|\right)\left\|I^{\prime}\left(u_{n}\right)\right\|-p^{-} I\left(u_{n}\right) \\
\geq & \left\langle I^{\prime}\left(u_{n}\right), u_{n}\right\rangle-p^{-} I\left(u_{n}\right) \\
= & M\left(\int_{0}^{T} \frac{\left|\dot{u}_{n}(t)\right|^{p(t)}}{p(t)} d t\right) \int_{0}^{T}\left|\dot{u}_{n}(t)\right|^{p(t)} d t-\int_{0}^{T}\left(\nabla V\left(t, u_{n}(t)\right), u_{n}(t)\right) d t \\
& -p^{-}\left[\widehat{M}\left(\int_{0}^{T} \frac{\left|\dot{u}_{n}(t)\right|^{p(t)}}{p(t)} d t\right)-\int_{0}^{T} V\left(t, u_{n}\right) d t\right] \\
\geq & p^{-} M\left(\int_{0}^{T} \frac{\left|\dot{u}_{n}(t)\right|^{p(t)}}{p(t)} d t\right) \int_{0}^{T} \frac{\left|\dot{u}_{n}(t)\right|^{p(t)}}{p(t)} d t-p^{-} \widehat{M}\left(\int_{0}^{T} \frac{\left|\dot{u}_{n}(t)\right|^{p(t)}}{p(t)} d t\right) \\
& +\int_{0}^{T}\left[p^{-} V\left(t, u_{n}(t)\right)-\left(\nabla V\left(t, u_{n}(t)\right), u_{n}(t)\right)\right] d t \\
\geq & \int_{0}^{T}\left[p^{-} V\left(t, u_{n}(t)\right)-\left(\nabla V\left(t, u_{n}(t)\right), u_{n}(t)\right)\right] d t \\
\geq & \int_{0}^{T} \frac{V\left(t, u_{n}(t)\right)}{\theta\left(\left|u_{n}(t)\right|\right)} d t-\int_{0}^{T} d(t) d t .
\end{aligned}
$$

Hence we have

$$
\int_{0}^{T} \frac{V\left(t, u_{n}(t)\right)}{\theta\left(\left|u_{n}(t)\right|\right)} d t \leq C_{3}
$$

Define

$$
f(s):=V(t, s x) .
$$

By $\left(V_{6}\right)$, we have

$$
f(s) \geq 0, \quad \forall s \geq \frac{L}{|x|} .
$$

Then deduce from $\left(V_{5}\right)$

$$
\begin{aligned}
f^{\prime}(s) & =\frac{1}{s}(\nabla V(t, s x), s x) \\
& \leq \frac{1}{s}\left(p^{-}-\frac{1}{\theta(s|x|)}\right) V(t, s x) \\
& =\frac{1}{s}\left(p^{-}-\frac{1}{\theta(s|x|)}\right) f(s)
\end{aligned}
$$

for all $s \geq \frac{L}{|x|}$. Let

$$
g(s):=f^{\prime}(s)-\frac{1}{s}\left(p^{-}-\frac{1}{\theta(s|x|)}\right) f(s) \leq 0 .
$$


By solving the above equation, we obtain

$$
f(s)=\left(\int_{\frac{L}{|x|}}^{s} \frac{g(r)}{r^{p^{-}} G(r|x|)} d r+\frac{f\left(\frac{L}{|x|}\right)|x|^{p^{-}}}{L^{p^{-}}} s^{p^{-}} G(s|x|)\right), \quad \forall s \geq \frac{L}{|x|},
$$

where

$$
G(|x|)=\exp \left(-\int_{L}^{|x|} \frac{1}{s \theta(s)} d s\right)
$$

which implies that

$$
f(s) \leq \frac{f\left(\frac{L}{|x|}\right)|x|^{p^{-}}}{L^{p^{-}}} s^{p^{-}} G(s|x|), \quad \forall s \geq \frac{L}{|x|}
$$

Therefore,

$$
V(t, x)=f(1) \leq \frac{V\left(t, \frac{L x}{|x|}\right)}{L^{p^{-}}}|x|^{p^{-}} G(|x|), \quad \forall|x| \geq L .
$$

By assumption $\left(V_{0}\right)$, we have

$$
V\left(t, \frac{L x}{|x|}\right) \leq \max _{|x| \leq L} a(|x|) b(t)
$$

for a.e. $t \in[0, T]$ and all $x \in \mathbb{R}^{N}$. Thus, we get

$$
V(t, x) \leq\left[\frac{|x|^{p^{-}}}{L^{p^{-}}} G(|x|)+1\right] \max _{|x| \leq L} a(|x|) b(t)
$$

for a.e. $t \in[0, T]$ and all $x \in \mathbb{R}^{N}$. Furthermore, by $\left(\theta_{2}\right)$, we obtain

$$
G(|x|) \rightarrow 0 \quad \text { as }|x| \rightarrow+\infty
$$

From $0<\frac{1}{\theta(\tau)}<p^{-}$, for $\tau>0$, we have

$$
\left(\tau^{p^{-}} G(\tau)\right)^{\prime}=\tau^{p^{-}-1} G(\tau)\left(p^{-}-\frac{1}{\theta(\tau)}\right)>0
$$

which implies that $\tau^{p^{-}} G(\tau)$ is increasing on $\tau$.

Combining (4.6) and (2.2), we get

$$
\begin{aligned}
\int_{0}^{T} V\left(t, u_{n}(t)\right) d t & \leq \int_{0}^{T}\left[\frac{\left|u_{n}(t)\right|^{p^{-}}}{L^{p^{-}}} G\left(\left|u_{n}(t)\right|\right)+1\right] \max _{|s| \leq L} a(|s|) b(t) d t \\
& \leq C_{4}\left\|u_{n}\right\|_{\infty}^{p^{-}} \int_{0}^{T} G\left(\left\|u_{n}\right\|_{\infty}\right) d t+C_{5} \\
& \leq C_{4} C_{0}^{p^{-}}\left\|u_{n}\right\|^{p^{-}} \int_{0}^{T} G\left(C_{0}\left\|u_{n}\right\|\right) d t+C_{5} .
\end{aligned}
$$


For $\forall u \in W_{T}^{1, p(t)}$, let

$$
\bar{u}=\frac{1}{T} \int_{0}^{T} u(t) d t \in \mathbb{R}^{N}, \quad \tilde{u}(t)=u(t)-\bar{u} .
$$

By $\left(M_{0}\right)$ and (2.3), we obtain

$$
\begin{aligned}
\widehat{M}\left(\int_{0}^{T} \frac{\left|\dot{u}_{n}(t)\right|^{p(t)}}{p(t)} d t\right) & =\int_{0}^{\int_{0}^{T} \frac{\left|\dot{u}_{n}(t)\right|^{p(t)}}{p(t)} d t} M(s) d s \\
& \geq \frac{m_{0}}{p^{+}} \int_{0}^{T}\left|\dot{u}_{n}(t)\right|^{p(t)} d t \\
& \geq \frac{m_{0}}{p^{+}}\left(\left\|\tilde{u}_{n}\right\|^{p^{-}}-1\right) .
\end{aligned}
$$

By (4.1), (4.7) and (4.8), we have

$$
\begin{aligned}
C_{1} & \geq I\left(u_{n}\right) \\
& =\widehat{M}\left(\int_{0}^{T} \frac{\left|\dot{u}_{n}(t)\right|^{p(t)}}{p(t)} d t\right)-\int_{0}^{T} V\left(t, u_{n}(t)\right) d t \\
& \geq \frac{m_{0}}{p^{+}}\left(\left\|\widetilde{u}_{n}\right\|^{p^{-}}-1\right)-C_{4} C_{0}^{p^{-}}\left\|u_{n}\right\|^{p^{-}} \int_{0}^{T} G\left(C_{0}\left\|u_{n}\right\|\right) d t-C_{5} .
\end{aligned}
$$

We claim that the sequence $\left\{u_{n}\right\}$ is bounded in $W_{T}^{1, p(t)}$. Suppose that is not the case. Passing to a subsequence if necessary, we may assume that

$$
\lim _{n \rightarrow+\infty}\left\|u_{n}\right\|=+\infty
$$

Set

$$
\omega_{n}=\frac{u_{n}}{\left\|u_{n}\right\|}=\frac{\bar{u}_{n}}{\left\|u_{n}\right\|}+\frac{\widetilde{u}_{n}}{\left\|u_{n}\right\|}=\bar{\omega}_{n}+\widetilde{\omega}_{n},
$$

then $\left\{\omega_{n}\right\}$ is bounded in $W_{T}^{1, p(t)}$ and $\left\|\omega_{n}\right\|=1$. Hence, up to a subsequence, we get

$$
\omega_{n} \rightarrow \omega \quad \text { weakly in } W_{T}^{1, p(t)}
$$

and

$$
\omega_{n} \rightarrow \omega \text { strongly in } C\left([0, T] ; \mathbb{R}^{N}\right) .
$$

By using Eqs. (4.6) and (4.10), one has

$$
\lim _{n \rightarrow+\infty} G\left(C_{0}\|u\|\right)=0
$$

Dividing both sides of (4.9) by $\left\|u_{n}\right\|^{p^{-}}$, by (4.10) and (4.11), we find that

$$
\lim _{n \rightarrow+\infty}\left\|\widetilde{\omega}_{n}\right\|=0 .
$$


So we deduce that $\omega=\bar{\omega} \in \mathbb{R}^{N}$ and $\omega \neq 0$. Thus

$$
\lim _{n \rightarrow+\infty} u_{n}(t)=+\infty
$$

for a.e. $t \in[0, T]$. By $\left(V_{7}\right)$, we get

$$
\lim _{\left|u_{n}\right| \rightarrow+\infty} \int_{0}^{T} \frac{V\left(t, u_{n}(t)\right)}{\theta\left(\left|u_{n}(t)\right|\right)} d t=+\infty .
$$

This contradicts (4.4). Therefore $\left\{u_{n}\right\}$ is bounded in $W_{T}^{1, p(t)}$. Similar to the proof of Lemma 3.1, we see that $I$ satisfies condition $(C)$.

Proof of Theorem 1.2 By saddle point theorem (see Theorem 4.6 in [37]), we only need to verify the following linking conditions:

$\left(I_{1}\right) I(u) \rightarrow+\infty$ as $\|u\| \rightarrow+\infty$ in $\widetilde{W}_{T}^{1, p(t)}$, where $\widetilde{W}_{T}^{1, p(t)}=\left\{u \in W_{T}^{1, p(t)} \mid \bar{u}=0\right\}$.

$\left(I_{2}\right) I(u) \rightarrow-\infty$ as $|u| \rightarrow+\infty$ in $\mathbb{R}^{N}$.

On one hand, by $\left(M_{0}\right),(2.3)$ and (4.7), for all $u \in \widetilde{W}_{T}^{1, p(t)}$, we have

$$
\begin{aligned}
I(u) & =\widehat{M}\left(\int_{0}^{T} \frac{|\dot{u}(t)|^{p(t)}}{p(t)} d t\right)-\int_{0}^{T} V(t, u(t)) d t \\
& =\int_{0}^{\int_{0}^{T} \frac{|\dot{u}(t)| p(t)}{p(t)} d t} M(s) d s-\int_{0}^{T} V(t, u(t)) d t \\
& \geq \frac{m_{0}}{p^{+}} \int_{0}^{T}|\dot{u}(t)|^{p(t)} d t-\int_{0}^{T} V(t, u(t)) d t \\
& \geq \frac{m_{0}}{p^{+}}\left(\|u\|^{p^{-}}-1\right)-C_{4} C_{0}^{p^{-}}\|u\|^{p^{-}} \int_{0}^{T} G\left(C_{0}\|u\|\right) d t-C_{5} \\
& =\|u\|^{p^{-}}\left[\frac{m_{0}}{p^{+}}\left(1-\frac{1}{\|u\| p^{-}}\right)-C_{4} C_{0}^{p^{-}} \int_{0}^{T} G\left(C_{0}\|u\|\right) d t\right]-C_{5} .
\end{aligned}
$$

Hence, by (4.11), we have

$$
I(u) \rightarrow+\infty \quad \text { as }\|u\| \rightarrow+\infty
$$

for all $u \in \widetilde{W}_{T}^{1, p(t)}$.

On the other hand, since $0<\frac{1}{\theta(|x|)}<p^{-}$, by $\left(V_{6}\right)$ and $\left(V_{7}\right)$, we have

$$
\begin{aligned}
I(u) & =-\int_{0}^{T} V(t, u(t)) d t \\
& \leq-\frac{1}{p^{-}} \int_{0}^{T} \frac{V(t, u(t))}{\theta(|u(t)|)} d t \\
& \rightarrow-\infty \text { as }|u| \rightarrow+\infty \text { in } \mathbb{R}^{N}
\end{aligned}
$$

Now, the proof of Theorem 1.2 is completed. 


\section{Funding}

This work is supported by the National Natural Science Foundation of China (31260098), Fundamental Research Funds for the Central Universities (31920180041, 31920190057), and the Fund for Talent Introduction of Northwest Minzu University (xbmuyjrc201907)

\section{Abbreviations}

Not applicable.

\section{Availability of data and materials}

Not applicable.

\section{Ethics approval and consent to participate}

The author contributed to each part of this study.

\section{Competing interests}

The author declares that he has no competing interests.

\section{Consent for publication}

The author read and approved the final version of the manuscript.

\section{Authors' contributions}

The author contributed solely to the writing of this paper. He read and approved the manuscript.

\section{Publisher's Note}

Springer Nature remains neutral with regard to jurisdictional claims in published maps and institutional affiliations.

Received: 31 March 2019 Accepted: 25 June 2019 Published online: 05 July 2019

\section{References}

1. Mawhin, J., Willem, M.: Critical Point Theory and Hamiltonian Systems. Springer, New York (1989)

2. Zhikov, V.V.: Averaging of functionals in the calculus of variations and elasticity. Math. USSR, Izv. 29, 33-66 (1987)

3. Ruzicka, M.: Electrorheological Fluids: Modeling and Mathematical Theory. Springer, Berlin (2002)

4. Radulescu, V.D., Zhang, B.L.: Morse theory and local linking for a nonlinear degenerate problem arising in the theory of electrorheological fluids. Nonlinear Anal., Real World Appl. 121, 336-369 (2015)

5. Chen, Y., Levine, S., Rao, M.: Variable exponent, linear growth functionals in image restoration. SIAM J. Appl. Math. 66, 1383-1406 (2006)

6. Karama, F., Sadik, K., Ziad, L.: A variable exponent nonlocal $p(x)$-Laplacian equation for image restoration. Comput. Math. Appl. 75, 534-546 (2018)

7. Fan, X.L., Zhao, D:: On the spaces $L^{p(x)}(\Omega)$ and $W^{m p(x)}(\Omega)$. J. Math. Anal. Appl. 263, 424-446 (2001)

8. Fan, X.L.: A Knobloch-type result for $p(t)$-Laplacian systems. J. Math. Anal. Appl. 282, 453-464 (2003)

9. Wang, X.J., Yuan, R.: Existence of periodic solutions for $p(t)$-Laplacian systems. Nonlinear Anal., Theory Methods Appl. $70,866-880(2009)$

10. Zhang, L., Tang, X.H.: Subharmonic solutions for some nonautonomous Hamiltonian systems with $p(t)$-Laplacian. Bull. Belg. Math. Soc. Simon Stevin 18, 385-400 (2011)

11. Zhang, L., Tang, X.H.: Periodic solutions for some nonautonomous p(t)-Laplacian Hamiltonian systems. Appl. Math. 58, 39-61 (2013)

12. Chen, P., Tang, X.H., Agarwal, R.: Infinitely many homoclinic solutions for nonautonomous $p(t)$-Laplacian Hamiltonian systems. Comput. Math. Appl. 63, 751-763 (2012)

13. Zhang, Z.H., Yuan, R.: Existence of two almost homoclinic solutions for $p(t)$-Laplacian Hamiltonian systems with a small perturbation. J. Appl. Math. Comput. 52, 173-189 (2016)

14. Zhang, Z.H., Xiang, T., Yuan, R.: Homoclinic solutions for $p(t)$-Laplacian-Hamiltonian systems without coercive conditions. Mediterr. J. Math. 13(4), 1589-1611 (2016)

15. An, Y.K., Ru, Y.F., Wang, F.L.: Existence of nonconstant periodic solutions for a class of second-order systems with $p(t)$-Laplacian. Bound. Value Probl. 2017, Article ID 170 (2017)

16. Chipot, M., Lovat, B.: Some remarks on non local elliptic and parabolic problems. Nonlinear Anal., Theory Methods Appl. 30, 4619-4627 (1997)

17. Gasinsk, L., Junior, J.R.S.: Multiplicity of positive solutions for an equation with degenerate nonlocal diffusion. Comput. Math. Appl. 78, 136-143 (2019)

18. Junior, J.R.S., Siciliano, G.: Positive solutions for a Kirchhoff problem with vanishing nonlocal term. J. Differ. Equ. 265 2034-2043 (2018)

19. Radulescu, V.D., Repovs, D.D.: Partial Differential Equations with Variable Exponents: Variational Methods and Qualitative Analysis. Monographs and Research Notes in Mathematics. CRC Press, Boca Raton (2015)

20. Cencelj, M., Radulescu, V.D., Repovs, D.D.: Double phase problems with variable growth. Nonlinear Anal. 177, 270-287 (2018)

21. Ho, K., Sim, I.: A-priori bounds and existence for solutions of weighted elliptic equations with a convection term. Adv. Nonlinear Anal. 6(4), 427-445 (2017)

22. Liu, Y., Zhao, J.M.: Abstract Hardy spaces with variable exponents. Nonlinear Anal. 167, $29-50$ (2018)

23. Simsen, J., Simsen, M., Zimmermann, A.: Study of ODE limit problems for reaction-diffusion equations. Opusc. Math. 38(1), 117-131 (2018)

24. Fan, X.L.: On nonlocal $p(x)$-Laplacian Dirichlet problems. Nonlinear Anal., Theory Methods Appl. 72, 3314-3323 (2010) 
25. Dai, G.W., Ma, R.Y.: Solutions for a $p(x)$-Kirchhoff type equation with Neumann boundary data. Nonlinear Anal., Real World Appl. 12, 2666-2680 (2011)

26. Vilasi, L.: Eigenvalue estimates for stationary $p(x)$-Kirchhoff problems. Electron. J. Differ. Equ. 2016, Article ID 186 (2016)

27. Allaoui, M., Ourraoui, A.: Existence results for a class of $p(x)$-Kirchhoff problem with a singular weight. Mediterr. J. Math. 13(2), 677-686 (2016)

28. Zhang, S.G.: Periodic solutions for a class of second order Hamiltonian systems with $p(t)$-Laplacian. Bound. Value Probl. 2016, Article ID 211 (2016)

29. Miyagaki, O.H., Souto, M.A.S.: Superlinear problems without Ambrosetti and Rabinowitz growth condition. J. Differ. Equ. 245(12), 3628-3638 (2008)

30. Alves, C.O., Liu, S.B.: On superlinear $p(x)$-Laplacian equations in $\mathbb{R}^{N}$. Nonlinear Anal., Theory Methods Appl. 73(8), 2566-2579 (2010)

31. Tang, C.L., Wu, X.P.: Periodic solutions for a class of new superquadratic second order Hamiltonian systems. Appl. Math. Lett. 34(2), 65-71 (2014)

32. Li, Y., Li, L.: Existence and multiplicity of solutions for $p(x)$-Laplacian equations in $\mathbb{R}^{N}$. Bull. Malays. Math. Sci. Soc. 12(1), 1-9 (2015)

33. Papageorgiou, N.S., Radulescu, V.D., Repovs, D.D.: Robin problems with a general potential and a superlinear reaction. J. Differ. Equ. 263, 3244-3290 (2017)

34. Wang, Z.Y., Xiao, J.Z:: On periodic solutions of subquadratic second order non-autonomous Hamiltonian systems, Appl. Math. Lett. 40, $72-77$ (2015)

35. Gasinski, L., Papageorgiou, N.S.: Existence and multiplicity of solutions for Neumann p-Laplacian type equations. Adv. Nonlinear Stud. 8, 843-870 (2008)

36. Li, S.J., Willem, M.: Applications of local linking to critical point theory. J. Math. Anal. Appl. 189, 6-32 (1995)

37. Rabinowitz, P.H.: Minimax Methods in Critical Point Theory with Applications to Differential Equations. CBMS, vol. 65. Am. Math. Soc., Providence (1986)

\section{Submit your manuscript to a SpringerOpen ${ }^{\circ}$ journal and benefit from:}

- Convenient online submission

- Rigorous peer review

- Open access: articles freely available online

- High visibility within the field

- Retaining the copyright to your article

Submit your next manuscript at $\boldsymbol{~ s p r i n g e r o p e n . c o m ~}$ 